\title{
Switch to Glutamate Receptor 2-Lacking AMPA Receptors Increases Neuronal Excitability in Hypothalamus and Sympathetic Drive in Hypertension
}

\author{
De-Pei Li, Hee Sun Byan, and Hui-Lin Pan \\ Center for Neuroscience and Pain Research, Division of Anesthesiology and Critical Care, The University of Texas M.D. Anderson Cancer Center, Houston, \\ Texas 77030
}

\begin{abstract}
Glutamatergic synaptic input in the hypothalamic paraventricular nucleus (PVN) plays a critical role in regulating sympathetic outflow in hypertension. GluR2-lacking AMPA receptors (AMPARs) are permeable to $\mathrm{Ca}^{2+}$, and their currents show unique inward rectification. However, little is known about changes in the AMPAR composition and its functional significance in hypertension. In this study, we found that AMPARmediated EPSCs (AMPAR-EPSCs) of retrogradely labeled spinally projecting PVN neurons exhibited a linear current-voltage relationship in Wistar-Kyoto (WKY) rats. However, AMPAR-EPSCs of labeled PVN neurons in spontaneously hypertensive rats (SHR) displayed inward rectification at positive holding potentials, which were not altered by lowering blood pressure with celiac ganglionectomy. Blocking GluR2lacking AMPARs with 1-naphthyl acetyl spermine (NAS) caused a greater reduction in the AMPAR-EPSC amplitude and firing activity of PVN neurons in SHR than in WKY rats. Furthermore, blocking NMDA receptors and inhibition of calpain or calcineurin abolished inward rectification of AMPAR-EPSCs of PVN neurons in SHR. The GluR2 protein level was significantly less in the plasma membrane but greater in the cytosolic vesicle fraction in SHR than in WKY rats. In addition, microinjection of NAS into the PVN decreased blood pressure and lumbar sympathetic nerve activity in SHR but not in WKY rats. Our study reveals that increased GluR2-lacking AMPAR activity of PVN neurons results from GluR2 internalization through NMDA receptor-calpain-calcineurin signaling in hypertension. This phenotype switch in synaptic AMPARs contributes to increased excitability of PVN presympathetic neurons and sympathetic vasomotor tone in hypertension.
\end{abstract}

\section{Introduction}

Essential (primary) hypertension is a prevalent clinical problem but the etiologies remain essentially unknown. The CNS, especially the hypothalamus (Yamori and Okamoto, 1969; Eilam et al., 1991), is critically involved in the development of essential hypertension by increasing sympathetic vasomotor tone (Anderson et al., 1989; Esler, 1995; Victor and Shafiq, 2008). The paraventricular nucleus (PVN) in the hypothalamus plays an important role in the regulation of sympathetic drive and blood pressure in hypertension (Allen, 2002; Li and Pan, 2007). The PVN presympathetic neurons control sympathetic outflow through projections to the intermediolateral cell column in the spinal cord and rostral ventrolateral medulla in the brainstem (Yamashita et al., 1984; Ranson et al., 1998; Li et al., 2003). Although the PVN normally is not essential in the regulation of the basal sympathetic vasomotor tone in normotensive conditions (Zahner and Pan, 2005; Li and Pan, 2007; Li et al., 2008), increased neuronal excitability in the PVN maintains elevated sympathetic

\footnotetext{
Received June 24, 2011; revised Oct. 25, 2011; accepted Nov. 7, 2011.

Author contributions: D.-P.L. and H.-L.P. designed research; D.-P.L. and H.S.B. performed research; D.-P.L. and H.S.B. analyzed data; D.-P.L. and H.-L.P. wrote the paper.

This study was supported by American Heart Association South Central Affiliate Grant 10GRNT3450003, National Institutes of Health Grant R01 HL077400, and the N.G. and Helen T. Hawkins Endowment (H.-L.P.).

Correspondence should be addressed to De-Pei Li or Hui-Lin Pan, Division of Anesthesiology and Critical Care, Unit 110, The University of Texas M.D. Anderson Cancer Center, 1515 Holcombe Boulevard, Houston, TX 77030. E-mail: dpli@mdanderson.org or huilinpan@mdanderson.org.

DOI:10.1523/JNEUROSCI.3222-11.2012

Copyright $\odot 2012$ the authors $\quad 0270-6474 / 12 / 320372-09 \$ 15.00 / 0$
}

drive in animal models of essential hypertension (Allen, 2002; Li and Pan, 2007). However, the synaptic mechanisms underlying increased PVN neuronal activity in hypertension are not fully known.

The AMPA receptors (AMPARs) mediate fast glutamatergic EPSCs in the CNS. Functional AMPARs are formed by heterotetrameric combinations of GluR1-GluR4 subunits, which determine the electrophysiological properties such as showing inward rectifying currents and permeability to $\mathrm{Ca}^{2+}$ (Dingledine et al., 1999; Mayer and Armstrong, 2004). The GluR2 subunit is a key determinant of biophysical properties of AMPARs because an arginine residue (R607) in the pore-lining region makes GluR2containing AMPARs impermeable to $\mathrm{Ca}^{2+}$ (Hollmann et al., 1991; Cull-Candy et al., 2006; Isaac et al., 2007). In contrast, GluR2-lacking AMPARs are highly permeable to $\mathrm{Ca}^{2+}$ and are voltage dependently blocked by intracellular polyamines (Bowie and Mayer, 1995; Donevan and Rogawski, 1995; Kamboj et al., 1995). Stimulation of NMDA receptors can induce GluR2 internalization in hippocampal neurons (Tigaret et al., 2006; Biou et al., 2008). Because NMDA receptor activity is increased in PVN presympathetic neurons in hypertension ( $\mathrm{Li}$ and Pan, 2007; Li et al., 2008), this may cause GluR2 internalization and a switch from GluR2-containing to GluR2-lacking AMPARs. Although GluR1GluR3 subunits of AMPARs are expressed in the PVN (Eyigor et al., 2001), little is known about changes in AMPAR compositions and their functional significance in hypertension.

In this study, we determined the changes in the activity of GluR2-lacking AMPARs and their role in increased excitability of 
PVN presympathetic neurons and sympathetic vasomotor tone in hypertension. We also determined the signaling mechanisms involved in increased activity of GluR2-lacking AMPARs in the PVN in hypertension. Our study reveals that the activity of GluR2-lacking AMPARs of PVN presympathetic neurons is increased in hypertension and that this synaptic plasticity results from GluR2 internalization mediated by the NMDA receptorcalpain-calcineurin signaling cascade. This new information greatly improves our understanding of the AMPAR plasticity and its role in increased excitability of PVN presympathetic neurons and sympathetic outflow in hypertension.

\section{Materials and Methods}

Experiments were performed using male spontaneously hypertensive rats (SHR) and age-matched Wistar-Kyoto (WKY) rats (13-15 weeks old; Taconic Farms). SHR is the most commonly used and bestcharacterized rat model of essential hypertension. The surgical procedures and experimental protocols were approved by the Animal Care and Use Committee of The University of Texas M.D. Anderson Cancer Center and conformed to the National Institutes of Health guidelines on the ethical use of animals. Blood pressure was measured daily for at least 1 week before the experiments by using a non-invasive tail-cuff system (IITC Life Science). The systolic arterial blood pressure (ABP) in 13week-old SHR $(205.5 \pm 10.6 \mathrm{mmHg}, n=47)$ was significantly higher than that of the age-matched WKY rats $(121.9 \pm 12.6 \mathrm{mmHg}, n=31)$.

Retrograde labeling of spinally projecting PVN neurons. We identified spinally projecting PVN neurons by using retrograde labeling, as described previously (Li et al., 2003; Li and Pan, 2007). Briefly, rats were anesthetized with $2-3 \%$ isoflurane in $\mathrm{O}_{2}$, and a limited dorsal laminectomy between T2 and T4 was performed to expose the spinal cord. FluoSpheres $(0.04 \mu \mathrm{m}$; Invitrogen) was pressure ejected bilaterally (Nanojector II; Drummond Scientific) using a glass pipette placed into the intermediolateral region of the spinal cord $(\sim 500 \mu \mathrm{m}$ from the midline and $\sim 500 \mu \mathrm{m}$ below the dorsolateral sulcus) in three to four separate $50 \mathrm{nl}$ injections. After injection, rats were treated prophylactically with an antibiotic $(5 \mathrm{mg} / \mathrm{kg}$ enrofloxacin, s.c., daily for $3 \mathrm{~d}$ ) and an analgesic ( $0.5 \mathrm{mg} / \mathrm{kg}$ buprenorphine, s.c., every $12 \mathrm{~h}$ for $3 \mathrm{~d})$. The rat was returned to its cage for 3-5 $\mathrm{d}$ to permit the FluoSpheres to be transported to the PVN.

Preparation of hypothalamic slices. Brain slices containing the PVN were prepared from the FluoSphere-injected rats, as described previously (Li et al., 2003, 2008). Briefly, the rats were anesthetized with $2 \%$ isoflurane and decapitated, and the brain was quickly removed and placed in ice-cold artificial CSF (aCSF; saturated with a mixture of $95 \% \mathrm{O}_{2}$ and 5\% $\mathrm{CO}_{2}$ ). The aCSF solution contained the following (in $\mathrm{mM}$ ): $124.0 \mathrm{NaCl}$, 3.0 KCl, 1.3 $\mathrm{MgSO}_{4}, 2.4 \mathrm{CaCl}_{2}, 1.4 \mathrm{NaH}_{2} \mathrm{PO}_{4}, 10.0$ glucose, and 26.0 $\mathrm{NaHCO}_{3}$. A tissue block containing the PVN was glued onto the stage of a vibrating microtome (Technical Products International). Coronal slices (300 $\mu \mathrm{m}$ thick) were cut as described previously (Li et al., 2003, 2008). The slices were then transferred to an incubation chamber containing aCSF continuously gassed with a mixture of $95 \% \mathrm{O}_{2}$ and $5 \% \mathrm{CO}_{2}$ at $34^{\circ} \mathrm{C}$ for at least $1 \mathrm{~h}$ before the electrophysiological recordings.

Electrophysiological recordings. Whole-cell patch-clamp recordings were performed in labeled PVN neurons in the hypothalamic slices. The recording chamber was continuously perfused $(3 \mathrm{ml} / \mathrm{min}$ ) with aCSF (saturated with $95 \% \mathrm{O}_{2}$ and $5 \% \mathrm{CO}_{2}$ ) at $34^{\circ} \mathrm{C}$, which was maintained by an in-line solution heater. The volume of the solution needed to fill the recording chamber was $\sim 1.0 \mathrm{ml}$. The labeled PVN neurons were identified using an upright microscope (BX51WI; Olympus) with a combination of epifluorescence illumination and differential interference contrast optics.

The recording electrode was pulled from borosilicate capillaries (1.2 $\mathrm{mm}$ outer diameter, $0.68 \mathrm{~mm}$ inner diameter; World Precision Instruments) by using a micropipette puller (P-97; Sutter Instruments). The resistance of the pipette was 3-7 M $\Omega$ when it was filled with an internal solution containing the following (in $\mathrm{mM}$ ): $140.0 \mathrm{~K}^{+}$gluconate, 2.0 $\mathrm{MgCl}_{2}, 0.1 \mathrm{CaCl}_{2}, 10.0$ HEPES, 1.1 EGTA, $0.3 \mathrm{Na}_{2}-\mathrm{GTP}$, and $2.0 \mathrm{Na}_{2}-$ ATP, adjusted to pH 7.25 (with $1 \mathrm{M} \mathrm{KOH}$ ) (270-290 mOsm). Signals were processed using a Multiclamp 700B amplifier (Molecular Devices), filtered at $1-2 \mathrm{kHz}$, and digitized at $20 \mathrm{kHz}$ using Digidata 1440 (Molec- ular Devices). To record the AMPA-EPSCs of PVN neurons, synaptic currents were evoked by electrical stimulation $(0.2 \mathrm{~ms}, 0.5 \mathrm{~mA}$, and 0.1 $\mathrm{Hz}$ ) through a bipolar tungsten electrode connected to a stimulator. The tip of the stimulation electrode was placed $\sim 150 \mu \mathrm{m}$ away from the recorded neuron on the ventral side of the PVN ( $\mathrm{Li}$ et al., 2003, 2008). The AMPAR-EPSCs were recorded at different holding potentials from -70 to $+50 \mathrm{mV}$ in the presence of $20 \mu \mathrm{m}$ bicuculline and $50 \mu \mathrm{M} \mathrm{AP}-5$ (for $5-10 \mathrm{~min}$ during the recording period). Spermine ( $0.1 \mathrm{~mm})$ was included in the internal solution for all the recordings. A current-voltage $(I-V)$ curve was created based on the amplitude of EPSCs recorded at different holding potentials. The spontaneous firing activity of labeled PVN neurons was recorded using the current-clamp mode without applying any holding currents (Li et al., 2008).

We also recorded postsynaptic AMPAR currents elicited by puff application of $100 \mu \mathrm{M}$ AMPA to labeled PVN neurons by using a Pressure System II (Toohey Company). The puff pipette ( $8-10 \mu \mathrm{m}$ tip diameter) was placed $50-100 \mu \mathrm{m}$ away from the recorded cells. Positive pressure was applied (4 psi, $100 \mathrm{~ms}$ duration) to eject AMPA onto the recorded cell to elicit AMPAR currents. All of the drugs were freshly prepared in aCSF before the experiments and delivered by using syringe pumps at the final concentrations indicated. AP-5, 6-cyano-7-nitroquinoxaline-2,3-dione (CNQX), bicuculline, tetrodotoxin (TTX), and AMPA were obtained from Ascent Scientific. 1-Naphthyl acetyl spermine (NAS) and FK506 were purchased from Sigma-Aldrich. Calpeptin was obtained from Tocris Bioscience.

Western immunoblotting. Rats were anesthetized with isoflurane and decapitated. Using the third ventricle as a reference, the PVN tissue $(\sim 0.5$ $\mathrm{mm}$ in diameter) spanning from 1.08 to $2.12 \mathrm{~mm}$ caudal to bregma was micropunched bilaterally on a cold plate under a dissection microscope. In each sample, PVN tissues from four rats were pooled to ensure that sufficient amounts of proteins were extracted. Subcellular fractionation was performed according to the procedures described previously (Park et al., 2009). Briefly, the tissue was homogenized in ice-cold buffer containing the following (in mM): 10 Tris, $\mathrm{pH} 7.4,1$ EDTA, $1 \mathrm{Na}_{3} \mathrm{VO}_{4}, 250$ sucrose, and the phosphatase and protease inhibitor cocktail (containing serine proteases, cysteine proteases, aspartic proteases, and metalloproteases; Sigma-Aldrich). The homogenate was centrifuged at $1000 \times g$ for $10 \mathrm{~min}$ at $4^{\circ} \mathrm{C}$ to remove nuclei and large debris. The supernatant was centrifuged at $20,000 \times g$ for $30 \mathrm{~min}$ at $4^{\circ} \mathrm{C}$ to separate the plasma membrane and cytosolic fractions. The cytosolic fraction sample was subsequently centrifuged at $150,000 \times g$ for $1 \mathrm{~h}$ at $4^{\circ} \mathrm{C}$, and the pellet was used as the cytosolic vesicle fraction (CVF). The plasma membrane fraction and CVF pellets were prepared by using the lysis buffer containing the following (in mM): 20 Tris, pH 7.6, 0.5\% NP-40, $250 \mathrm{NaCl}, 3$ EDTA, 3 EGTA, 2 DTT, and the protease inhibitor cocktail. The samples were incubated for $30 \mathrm{~min}$ at $4^{\circ} \mathrm{C}$ and then centrifuged at $12,000 \times g$ for $15 \mathrm{~min}$ at $4^{\circ} \mathrm{C}$. The supernatant was collected and the protein concentration was determined using the Lowry protein assay. For Western blotting, $50 \mu \mathrm{g}$ proteins were separated by $10 \%$ SDS-PAGE and transferred to a PVDF membrane (Immobilon P; Millipore). The immunoblots were probed with a rabbit anti-GluR1 antibody (1:1000; Millipore), a rabbit antiGluR2 antibody (1:1000; Millipore), and an anti- $\beta$-actin antibody (1: 5000; Cell Signaling Technology). The specificity of anti-GluR1 and antiGluR2 antibodies have been shown previously (Park et al., 2009). ECL kit (GE Healthcare) was used to detect the protein bands, and the band density was analyzed with AIS software (Imaging Research). The amounts of GluR1 and GluR2 proteins were quantified by normalizing the optical density of their protein band to that of $\beta$-actin (serving as a protein loading control). The mean values of GluR1 and GluR2 amounts in WKY rats were considered to be 1 .

In vivo recording of lumbar sympathetic nerve activity, $A B P$, and heart rate. Rats were initially anesthetized using $2 \%$ isoflurane in $\mathrm{O}_{2}$ before intraperitoneal injection of a mixture of $\alpha$-chloralose $(60-75 \mathrm{mg} / \mathrm{kg}$ ) and urethane $(800 \mathrm{mg} / \mathrm{kg})$. The depth of anesthesia level was confirmed before surgery by the absence of both corneal reflexes and paw-withdrawal responses to a noxious pinch. The trachea was cannulated for mechanical ventilation using a rodent ventilator with $100 \% \mathrm{O}_{2}$. The ABP was monitored with a pressure transducer through a catheter placed into the left femoral artery. Heart rate (HR) was counted by triggering from the pul- 
satile blood pressure. The right femoral vein was cannulated for intravenous administration of supplemental doses of $\alpha$-chloralose and urethane to maintain an adequate depth of anesthesia. A small branch of the left lumbar postganglionic sympathetic nerve was isolated under an operating microscope through a retroperitoneal incision. The lumbar sympathetic nerve was cut distally to ensure that afferent activity was not recorded. The nerve was then immersed in mineral oil and placed on a stainless steel recording electrode (Li and Pan, 2007; Ye et al., 2011). The nerve signal was amplified and bandpass filtered $(100-3000 \mathrm{~Hz})$ by an alternating current amplifier (model P511; Grass Instruments), and the lumbar sympathetic nerve activity (LSNA) was monitored using an audio amplifier (Grass Instruments). The LSNA and ABP were recorded using a 1401PLUS analog-to-digital converter and Spike2 system (Cambridge Electronic Design). The level of background electrical noise was determined by suppressing the LSNA via injection of phenylephrine $(20 \mu \mathrm{g} / \mathrm{kg}$, i.v.) before the rats were killed and $5 \mathrm{~min}$ after the rats were killed by an overdose of pentobarbital sodium (200 $\mathrm{mg} / \mathrm{kg}$, i.v.) at the end of each experiment. The respective electrical noise levels were subtracted from the integrated LSNA values, and the percentage change in LSNA from baseline was calculated.

PVN microinjections. For PVN microinjections, rats were placed in a stereotactic frame and the brain was exposed at the level of the hypothalamus. A glass microinjection pipette (tip diameter, $20-30 \mu \mathrm{m}$ ) was advanced into the PVN using the following stereotactic coordinates: 1.6-2.0 $\mathrm{mm}$ caudal to bregma, $0.5 \mathrm{~mm}$ lateral to the midline, and $7.0-7.5 \mathrm{~mm}$ ventral to the dura. The injection sites of the PVN were first confirmed by the depressor responses to microinjection of 5.0 nmol GABA ( $20 \mathrm{nl}, 250 \mathrm{~mm}$ ). The microinjection was done by using a calibrated microinjection system (Nanojector II; Drummond Scientific) and monitored using an operating microscope. GABA microinjections were separated by $10-15 \mathrm{~min}$ intervals to allow for recovery of the depressor response. The PVN vasomotor site was considered to have been located when the GABA injection decreased the mean ABP by at least $10 \mathrm{mmHg}$ ( $\mathrm{Li}$ and Pan, 2007; Ye et al., 2011). The stereotactic coordinates at which the previous GABA microinjection elicited the greatest depressor responses were used in the same rat for the subsequent microinjection of NAS. After microinjection of the drugs, the glass pipette was left in place for 1-2 min to ensure adequate delivery of the drug to the injection site. The location of the pipette tip and diffusion of the drugs in the PVN were determined by including $5 \%$ FluoSpheres $(0.04 \mu \mathrm{m}$; Invitrogen) in the injection solution. The rat brain was removed rapidly at the end of the experiment and fixed in $10 \%$ buffered Formalin solution overnight. Frozen coronal sections ( $40 \mu \mathrm{m}$ thick) were cut on a freezing microtome and mounted on slides. Rhodamine-labeled fluorescent regions were identified using an epifluorescent microscope and plotted on standardized sections from the Paxinos and Watson (1998) atlas. Rats were not included for data analysis if they had one misplaced microinjection outside the PVN.

Celiac ganglionectomy and blood pressure measurement with telemetry. Surgery was performed aseptically in SHR anesthetized with $2 \%$ isoflurane in $\mathrm{O}_{2}$. The celiac ganglion area was exposed through a midline laparotomy. The bifurcations of the superior mesenteric artery and celiac plexus were identified, and the celiac ganglion was located within the area near the superior mesenteric artery and celiac artery. For rats undergoing celiac ganglionectomy (CGx), the celiac plexus and all visible nerves were dissected under a surgical microscope and removed by stripping. In sham control rats, the celiac ganglion plexus was exposed but not disturbed.

To measure blood pressure in SHR, the Milar catheter of the telemetry system (Telemetry Research) was inserted into the abdominal aorta below the bifurcation of the renal arteries (Ye et al., 2011). The ABP signal was feed into the transmitter, which was implanted in the abdominal cavity and attached with a suture to the peritoneum. The abdomen was closed in two layers with interrupted sutures. The rats were housed singly, and buprenorphine and enrofloxacin were given for $3 \mathrm{~d}$ after the surgery. The blood pressure signal was monitored in conscious rats through the receiver, and the data were collected every $2 \mathrm{~d}$ and analyzed with a data acquisition system (LabChart; AD Instruments). Two weeks after CGx or sham surgery, rats were used for brain slice recordings.

Data analysis. Data are presented as means \pm SEM. The firing rate, AMPAR currents, and AMPAR-EPSCs were analyzed offline by Clampfit (Molecular Devices). The junction potential was corrected offline based on the composition of the internal and external solution used for the recordings. The LSNA, ABP, and HR were analyzed using the Spike2 software program. LSNA was rectified and integrated offline after subtracting the background noise. Control values were obtained by averaging the signal over a $60 \mathrm{~s}$ period immediately before each treatment. Response values after each intervention were averaged over $30 \mathrm{~s}$ when the maximal responses occurred. The Student's $t$ test was used for comparisons of two datasets. For comparisons of more than two datasets, the 


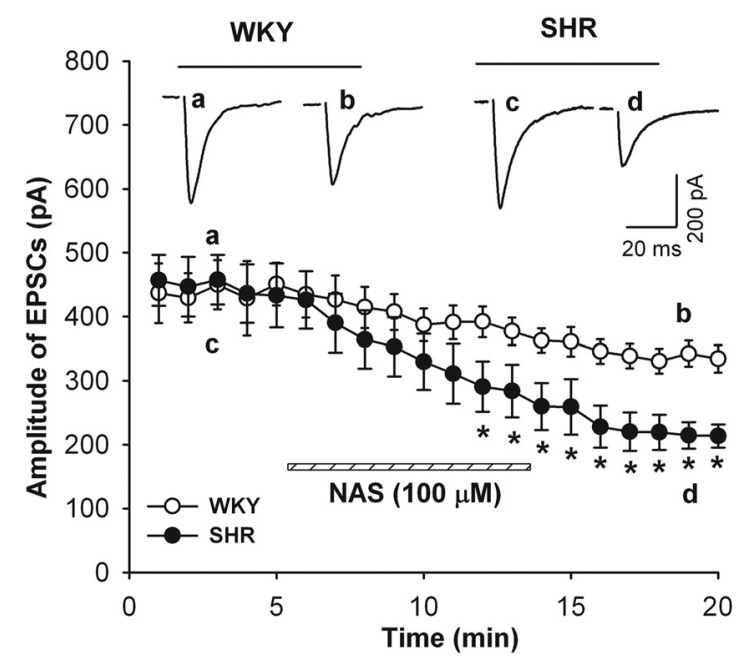

Figure 2. Contribution of GluR2-lacking AMPARs to AMPA-EPSCs in spinally projecting PVN neurons in SHR. Effect of bath application of NAS $(100 \mu \mathrm{m})$ on the amplitude of AMPAR-EPSCs in labeled PVN neurons in WKY rats $(n=8)$ and SHR $(n=9)$. Inset, Original traces show evoked AMPAR-EPSCs before and after NAS application in $\operatorname{WKY}(\boldsymbol{a}, \boldsymbol{b})$ and SHR $(\boldsymbol{c}, \boldsymbol{d})$. Data are presented as mean \pm SEM. ${ }^{*} p<0.05$ compared with control value before NAS application.

repeated-measures ANOVA with Dunnett's test or two-way ANOVA with Bonferroni's post hoc test was performed to compare responses within or between experimental groups, respectively. $p<0.05$ was considered statistically significant.

\section{Results}

Contribution of GluR2-lacking AMPARs to AMPAR-EPSCs and firing activity of spinally projecting PVN neurons in SHR Because of the voltage-dependent block by intracellular polyamines, GluR2-lacking AMPARs are highly permeable to $\mathrm{Ca}^{2+}$ and exhibit inward rectification at positive holding potentials (Bowie and Mayer, 1995; Isaac et al., 2007). On the basis of this unique biophysical feature, we assessed the $I-V$ relationship of AMPAR-EPSCs of spinally projecting PVN neurons in the presence of $50 \mu \mathrm{M} \mathrm{AP}-5$ and $20 \mu \mathrm{M}$ bicuculline. To compensate for a possible loss of endogenous polyamines from intracellular dialysis during whole-cell recordings, we included $0.1 \mathrm{~mm}$ spermine in the intracellular solution (Vikman et al., 2008). The $I-V$ curve of AMPAR-EPSCs in labeled PVN neurons in WKY rats displayed a linear $I-V$ relationship from -70 to $+50 \mathrm{mV}$. However, the AMPAR-EPSCs displayed a profound inward rectification at holding potentials from 10 to $50 \mathrm{mV}$ in SHR (Fig. $1 A, B)$. The rectification index (RI; $\left.I_{+30 \mathrm{mV}} / I_{-50 \mathrm{mV}}\right)$ in labeled PVN neurons from WKY rats $(0.87 \pm 0.11, n=9)$ was significantly greater than that in $\operatorname{SHR}(0.34 \pm 0.06, n=10, p<0.05$; Fig. $1 C)$. Treatment of the brain slices with $100 \mu \mathrm{M}$ NAS, a synthetic analog of Joro spider toxin that selectively blocks GluR2-lacking AMPARs (Blaschke et al., 1993; Vikman et al., 2008), converted the $I-V$ relationship of AMPAR-EPSCs of PVN neurons of SHR from inward rectifying to linear $(n=8$; Fig. $1 D)$. NAS treatment significantly reduced the conductance of AMPAR-EPSCs of these neurons in SHR (Fig. $1 E$ ).

We next determined the contribution of GluR2-lacking AMPARs to AMPAR-EPSCs in labeled PVN neurons in both WKY and SHR by using NAS. Bath application of $100 \mu \mathrm{M}$ NAS for $10 \mathrm{~min}$ progressively decreased the amplitude of AMPAR-EPSCs in WKY rats from $430.8 \pm 33.5$ to $334.2 \pm 21.6 \mathrm{pA}$ (21.9 $\pm 2.5 \%$ reduction, $n=8)$. In SHR, NAS caused a much larger reduction in the amplitude of AMPAR-EPSCs from $413.0 \pm 49.8$ to $220.5 \pm 30.3 \mathrm{pA}$ (53.1 $\pm 4.8 \%$ reduction, $n=9$; Fig. 2 ) 10 min after NAS application.
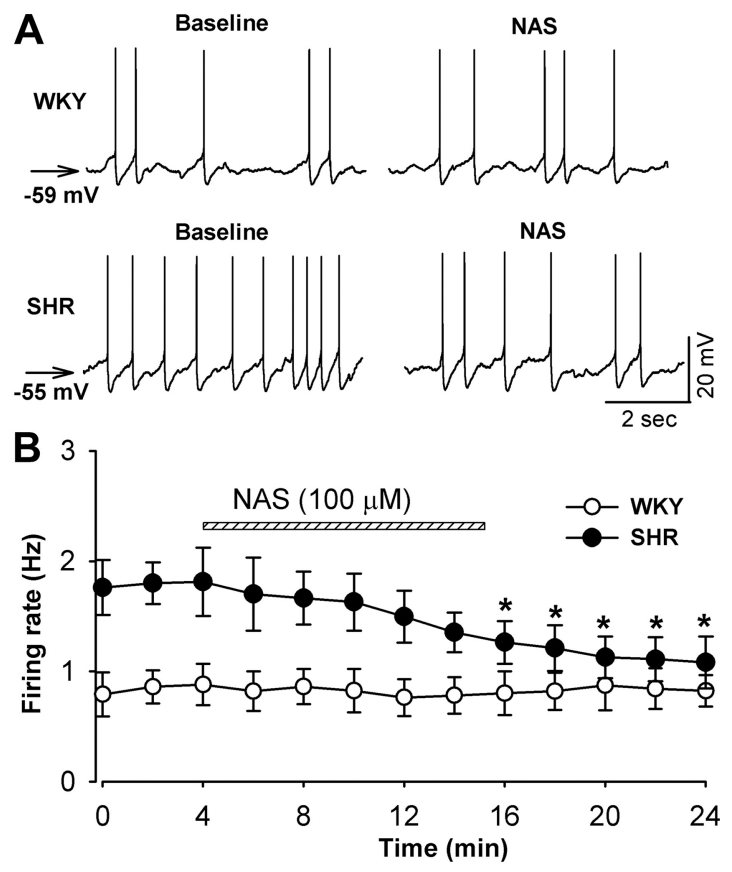

Figure 3. Blocking GluR2-lacking AMPARs reduces the firing activity of spinally projecting PVN neurons in SHR. A, Representative recordings show that NAS (100 $\mu \mathrm{m})$ did not change the firing rate of a labeled PVN neuron in a WKY rat but decreased the firing activity of a labeled PVN neuron in an SHR. $B$, Summary data show the effect of NAS $(100 \mu \mathrm{m})$ on the firing rate of labeled PVN neurons in WKY rats $(n=7)$ and SHR $(n=8)$. Data presented as means \pm SEM. ${ }^{*} p<0.05$ compared with the control value before NAS application.

We then determined the role of GluR2-lacking AMPARs in increased firing activity of spinally projecting PVN neurons in SHR. We compared the effect of NAS on the firing rate of labeled PVN neurons in SHR and WKY rats. The baseline spontaneous firing rate of PVN neurons was $1.8 \pm 0.3 \mathrm{~Hz}(n=8)$ in SHR, which was significantly higher than that $(0.8 \pm 0.3 \mathrm{~Hz}, n=7, p<$ $0.05)$ in WKY rats. Furthermore, the membrane potential of labeled PVN neurons was more depolarized in SHR $(-57.2 \pm 1.2$ $\mathrm{mV})$ than in WKY rats $(-59.5 \pm 1.5 \mathrm{mV}, n=8, p>0.05)$. Bath application of $100 \mu \mathrm{M}$ NAS for 10 min significantly decreased the firing rate of labeled PVN neurons in SHR $(n=8, p<0.05$; Fig. $3)$. However, NAS failed to significantly decrease the firing activity of labeled PVN neurons in WKY rats $(n=7, p>0.05$; Fig. 3$)$.

Role of GluR2-lacking AMPARs in the control of sympathetic vasomotor tone in SHR

To determine the role of GluR2-lacking AMPARs in the PVN in the control of sympathetic vasomotor tone in hypertension, we examined the effect of bilateral microinjection of NAS into the PVN on LSNA and ABP in WKY and SHR. The basal LSNA, ABP, and HR were significantly higher in SHR than in WKY rats. Bilateral microinjection of NAS (1 mM in $50 \mathrm{nl})$ into the PVN significantly decreased the LSNA, ABP, and HR by $17.6 \pm 5.4 \%, 30.8 \pm 6.6 \mathrm{mmHg}$, and $29.5 \pm 6.8 \mathrm{bpm}$, respectively, in SHR ( $n=9$; Fig. 4$)$. However, bilateral microinjection of NAS into the PVN failed to significantly alter LSNA, ABP, and HR in WKY rats ( $n=7$; Fig. 4$)$.

\section{Switch to GluR2-lacking AMPARs is not attributable to high} blood pressure and hyperactivity of PVN neurons in SHR

To further determine whether increased GluR2-lacking AMPAR activity in PVN neurons is a secondary change to high blood pressure in SHR, we examined the $I-V$ relationship of AMPAREPSCs of PVN neurons in SHR subjected to CGx (Ye et al., 2011). 

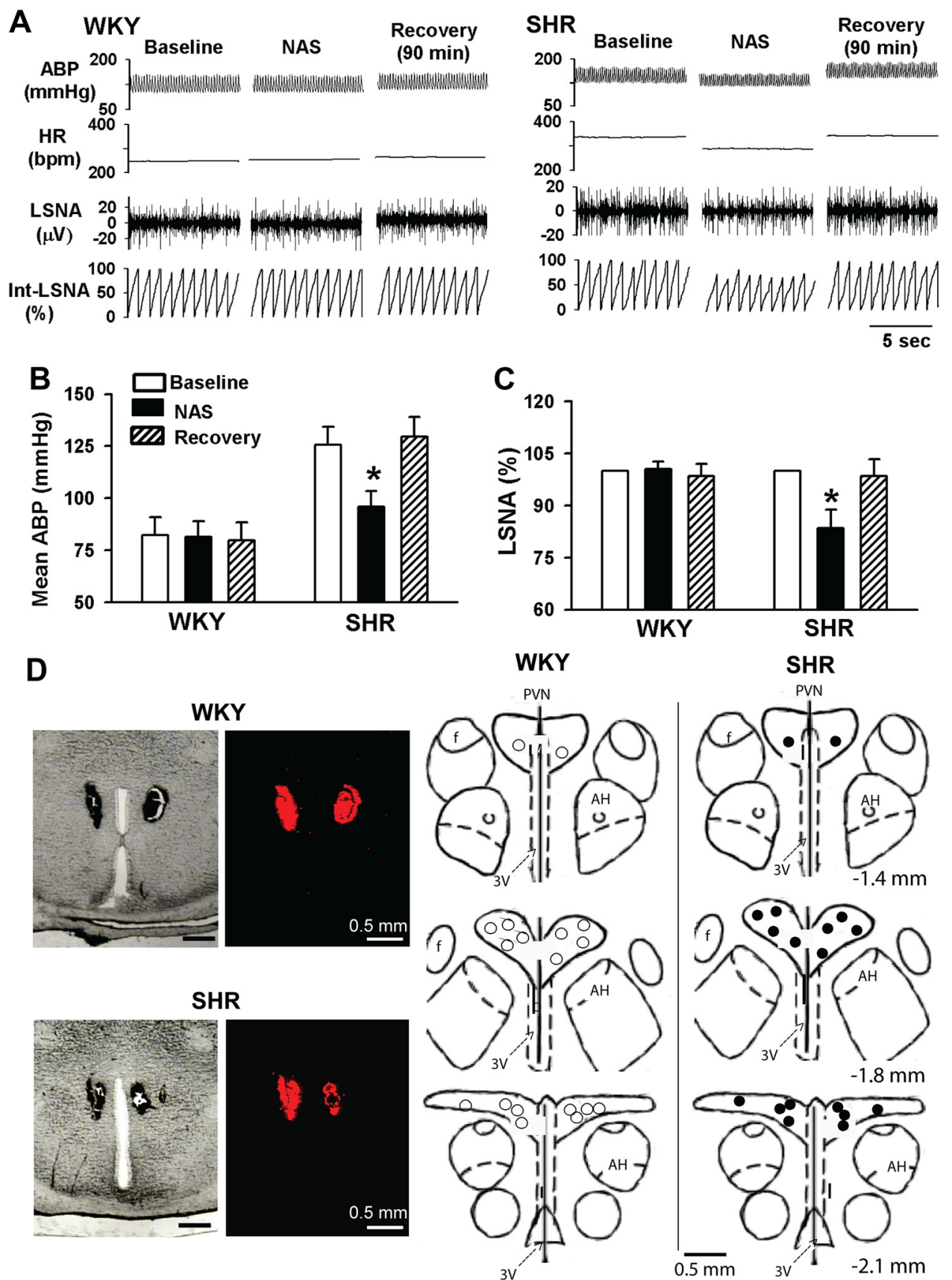

Figure 4. Responses of ABP, HR, and LSNA to microinjection of NAS into the PVN in WKY and SHR. A, Representative recordings depict that the differential effect of microinjection of NAS (1 mM, $50 \mathrm{nl}$ ) into the PVN on ABP, HR, and LSNA in one SHR and one WKY rat. $\boldsymbol{B}, \boldsymbol{C}$, Summary data show changes in mean ABP $(\boldsymbol{B})$ and LSNA $(\boldsymbol{C})$ in response to microinjection of NAS into the PVN in WKY ( $n=$ 7) and SHR $(n=9)$. D, Original photomicrographs (left) depicting the PVN injection site in a coronal brain slice of a WKY rat and an SHR, and schematic drawings (right) show the locations of NAS microinjection sites in WKY (O) and SHR (). Data presented as means \pm SEM. ${ }^{*} p<0.05$ compared with the corresponding baseline control. AH, Anterior hypothalamus; $3 \mathrm{~V}$, the third ventricle.

CGx, but not sham surgery, significantly lowered the mean ABP in SHR for at least 2 weeks (Fig. 5A). The $I-V$ relationship of evoked AMPAR-EPSCs of labeled PVN neurons still displayed an inward rectification at positive holding potentials (Fig. $5 A$ ). The RI values in SHR subjected to CGx was similar to SHR that received sham surgery $(0.39 \pm 0.07, n=9$ vs $0.35 \pm 0.06, n=8 ; p>0.05)$.

The firing activity of PVN presympathetic neurons is increased in SHR (Li and Pan, 2006; Li et al., 2008). To determine whether increased GluR2-lacking AMPAR activity results from hyperactivity of PVN neurons, we inhibited the firing activity of PVN neurons in SHR with $1 \mu \mathrm{M}$ TTX. Puff application of $100 \mu \mathrm{M}$ AMPA elicited inward currents in neg- ative holding potentials and outward currents in positive holding potentials (Fig. 5B). The $I-\mathrm{V}$ curve of AMPAR currents of labeled PVN neurons displayed an inward rectification at holding potentials (from 10 to $50 \mathrm{mV}$; Fig. $5 B$ ). Bath application of $20 \mu \mathrm{M}$ CNQX blocked the AMPAR currents elicited by puff AMPA application. The $I-V$ curve of AMPAR currents of labeled PVN neurons in SHR still displayed an inward rectification at positive holding potentials after treatment with $1 \mu \mathrm{M}$ TTX for $1-2 \mathrm{~h}$ (Fig. $5 B$ ). The RI value of the AMPAR currents of labeled PVN neurons in SHR did not differ significantly between TTX-treated and vehicle-treated brain slices $(0.35 \pm 0.02, n=8$ vs $0.36 \pm 0.06, n=9 ; p>0.05)$. 

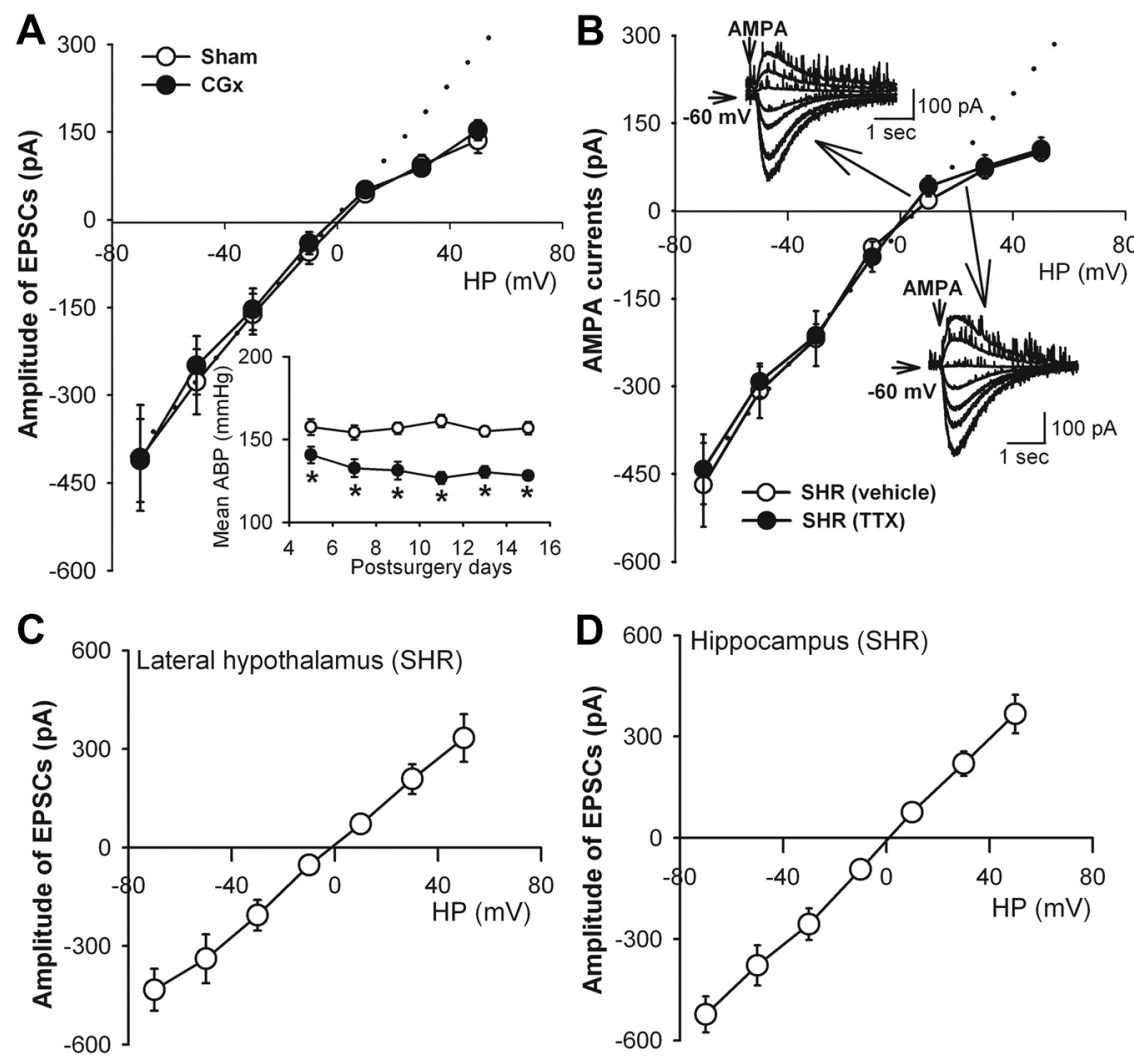

Figure 5. Lack of effect of reduction in blood pressure and inhibition of neuronal activity on the inward rectification of AMPAR-EPSCs of spinally projecting PVN neurons in SHR. $A, I-V$ relationship curves show inward rectification of AMPAR-EPSCs at positive potentials in the PVN neurons in SHR subjected to CGx $(n=9)$ or sham surgery $(n=8)$. Inset, Mean ABP recorded with the radiotelemetry system in conscious SHR subjected to $C G$ or sham surgery $\left(n=7\right.$ rats in each group). ${ }^{*} p<0.05$ compared with SHR receiving sham surgery. $\boldsymbol{B}$, Currents induced by puff AMPA application displayed an inward rectification at positive potentials in labeled PVN neurons of SHR rats treated with $1 \mu \mathrm{m} \Pi \mathrm{TX}(n=8)$ or vehicle $(n=9)$. Inset, Original traces show that puff AMPA-induced currents at holding potentials (HP) from -70 to $50 \mathrm{mV}$ of PVN neurons treated with TTX or vehicle. $C, D, I-V$ relationships of evoked AMPAR-EPSCs of neurons in the lateral hypothalamus $(n=8)$ and hippocampus $(n=9)$ in SHR. Dotted lines show linearity of the $I-V$ curves.

Additionally, to determine whether increased GluR2-lacking AMPAR activity in SHR is a global phenomenon in the brain, we determined the $I-V$ relationship of AMPAR-EPSCs of neurons in the lateral hypothalamus $(n=8)$ and hippocampus $(n=9)$ in SHR. The AMPAR-EPSCs of these neurons in the lateral hypothalamus and hippocampus displayed a linear $I-V$ relationship (Fig. 5C,D).

\section{GluR1 and GluR2 protein levels in the membrane and cytosolic fractions in the PVN in SHR}

To determine the subcellular distribution of GluR1 and GluR2 proteins in the PVN in WKY and SHR, we used Western immunoblotting to measure the protein amounts of GluR1 and GluR2 in the plasma membrane and cytosolic vesicle fractions. The GluR2 level in the plasma membrane fraction was significantly lower in the PVN in SHR than in WKY rats, whereas the GluR2 protein level in the cytosolic vesicle fraction was significantly higher in SHR than in WKY rats (Fig. 6A, B). However, the protein amounts of GluR1 subunit in the plasma membrane and cytosolic vesicle fraction in the PVN did not differ significantly between SHR and WKY rats (Fig. 6A,C).

\section{Signaling mechanisms underlying increased GluR2-lacking} AMPAR activity in PVN neurons in SHR

Activation of NMDA receptors induces internalization of GluR2 in cultured hippocampal neurons (Beattie et al., 2000; Biou et al., 2008). Because the NMDA receptor activity is upregulated in PVN presympathetic neurons in SHR ( $\mathrm{Li}$ and Pan, 2007; Li et al., 2008), we determined the role of NMDA receptors in increased activity of GluR2-lacking AMPARs in PVN neurons in SHR. The brain slices from SHR were incubated with solution containing the specific NMDA receptor antagonist AP-5 $(50 \mu \mathrm{M})$ for $1-2 \mathrm{~h}$. AP-5 treatment eliminated the inward rectification of AMPAREPSCs at positive potentials of labeled PVN neurons in SHR (Fig. 7A). Furthermore, AP-5 treatment normalized the RI of labeled PVN neurons in SHR $(0.75 \pm 0.13, n=8)$ to that seen in normotensive WKY rats.

NMDA receptor stimulation can activate the $\mathrm{Ca}^{2+}$-dependent protein phosphatase calcineurin to induce GluR1 endocytosis in cultured hippocampal neurons (Beattie et al., 2000). We next determined the role of calcineurin in increased activity of GluR2-lacking AMPARs in labeled PVN neurons in SHR. We incubated the hypothalamic slices from SHR with 1 $\mu \mathrm{M}$ FK506, a selective calcineurin inhibitor (Liu, 1993), for $1-2 \mathrm{~h}$. The $I-V$ curve of AMPAR-EPSCs displayed a linear relationship after FK506 treatment (Fig. $7 B)$. The RI of labeled PVN neurons was significantly greater in slices treated with FK506 $(0.82 \pm 0.12, n=9)$ than in vehicle-treated slices $(0.34 \pm 0.07, n=$ 7, $p<0.05$; Fig. 7D).

Calpain is a family of $\mathrm{Ca}^{2+}$-activated cysteine proteases (Croall and DeMartino, 1991; Molinari and Carafoli, 1997). NMDA receptor stimulation can increase calpain activity (Adamec et al., 1998; Hewitt et al., 1998) to potentiate calcineurin activity by converting calcineurin A subunit to the active form (Wu et al., 2004; Shioda et al., 2006). Thus, we determined whether calpain activity is involved in increased activity of GluR2-lacking AMPARs in PVN neurons in SHR. The hypothalamic slices from SHR were incubated with a selective membrane-permeable calpain inhibitor, calpeptin $(100 \mu \mathrm{M})$ (Tsujinaka et al., 1988) for $1-2 \mathrm{~h}$. The $I-V$ curve of evoked AMPAR-EPSCs of labeled PVN neurons in SHR showed a linear relationship after calpeptin treatment (Fig. 7C). The RI of PVN neurons was significantly greater in brain slices treated with calpeptin $(0.81 \pm 0.07, n=9)$ than in slices treated with vehicle $(0.36 \pm 0.04, n=8, p<0.05$; Fig. $7 D)$.

\section{Discussion}

Increased sympathetic outflow represents an important mechanism underlying the development and maintenance of essential hypertension (Judy et al., 1976; Anderson et al., 1989; Li and Pan, 2007). Previous studies have shown that the hypothalamus is critically involved in the elevated sympathetic outflow and ABP associated with hypertension. In this regard, transection of the brain caudal to the hypothalamus reduces ABP in SHR, but not in normotensive WKY rats (Yamori and Okamoto, 1969). Also, transplantation of embryonic hypothalamic tissues of SHR into the brain of adult normotensive rats results in the development of hypertension (Eilam et al., 1991). The hypothalamic PVN is an integrating center for regulation of neuroendocrine, cardiovas- 
cular, and other physiological functions (Swanson and Sawchenko, 1983). The hyperexcitability of PVN presympathetic neurons can directly and indirectly influence the sympathetic outflow and ABP in hypertension (Allen, 2002; Li and Pan, 2007; Li et al., 2008). We have shown that the glutamatergic input in the PVN is augmented and leads to heightened sympathetic vasomotor tone in SHR (Li and Pan, 2007; Li et al., 2008). However, it is not clear whether changes in the AMPAR composition contribute to glutamatergic synaptic plasticity in the PVN during the development of hypertension.

In the present study, we provide new evidence showing that the GluR2-lacking AMPAR activity is increased and this AMPAR phenotype switch contributes to elevated firing activity of spinally projecting PVN neurons in SHR. The unique biophysical property of GluR2-lacking AMPAR currents is the inward rectification because of the voltage-dependent block by internal polyamines (Bowie and Mayer, 1995; Koh et al., 1995). We found that AMPAR-mediated EPSCs displayed inward rectification at positive holding potentials in spinally projecting PVN neurons in SHR. Also, blocking GluR2lacking AMPARs with NAS produced a significantly greater reduction in the amplitude of AMPAR-EPSCs in PVN neurons in SHR than in WKY rats. Furthermore, NAS significantly decreased the firing activity of PVN neurons in SHR but not in WKY rats. Although blocking GluR2-lacking AMPARs slightly decreased the amplitude of AMPAR-EPSCs of PVN neurons, it had no significant effect on the firing activity of these neurons in WKY rats. It seems that low levels of GluR2-lacking AMPARs do not play a significant role in the regulation of the excitability of spinally projecting PVN neurons under the normotensive condition. Our data suggest that increased activity of GluR2-lacking AMPARs contributes significantly to maintaining elevated firing activity of PVN presympathetic neurons in hypertension.

Interestingly, we observed that the AMPAR-EPSCs of neurons in the lateral hypothalamus and hippocampus displayed a linear $I-V$ relationship, suggesting that increased GluR2-lacking AMPAR activity does not occur in all brain regions in SHR. It has been shown that $10 \mu \mathrm{M}$ NAS can block GluR2-lacking AMPARs in cultured hippocampal neurons (Koike et al., 1997). However, higher NAS concentrations $(100-250 \mu \mathrm{M})$ are used to block GluR2-lacking AMPARs in the tissue slice preparation (Noh et al., 2005; Vikman et al., 2008) because a higher drug concentration is required to reach an effective concentration to the cells recorded deep in the tissue slices. Membrane depolarization to $-20 \mathrm{mV}$ and above can relieve the NAS (3 $\mu \mathrm{M})$ block of GluR2-lacking AMPARs (Koike et al., 1997). In our study, the membrane potential of labeled PVN neurons in SHR was between -50 and $-60 \mathrm{mV}$ and may not reach the depolarizing level to relieve the blocking effect of $100 \mu \mathrm{M}$ NAS on GluR2-lacking AMPARs in these neurons. GluR2-lacking AMPARs are calcium permeable (Hollmann et al., 1991; Keller et al., 1992) and associated with a larger channel conductance than AMPARs assembled by a
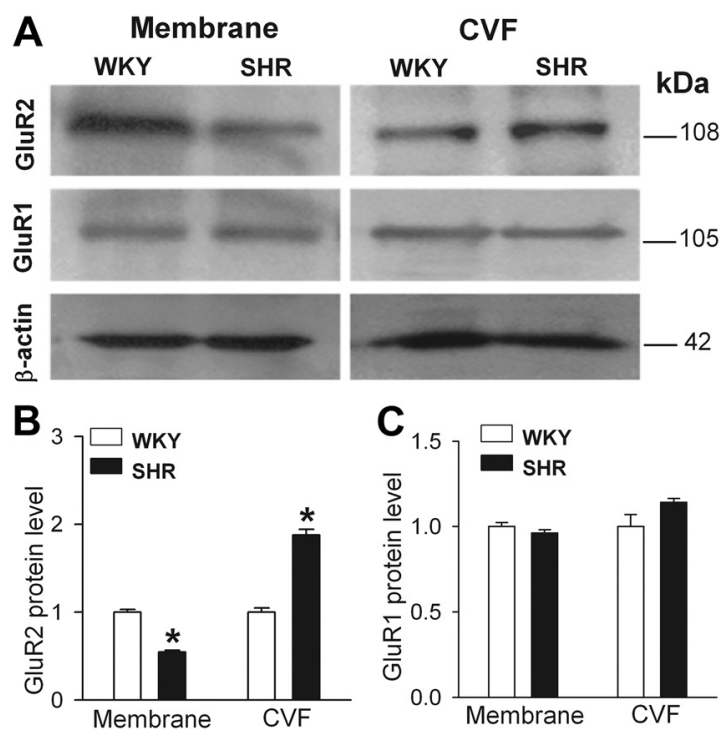

Figure 6. Subcellular distribution of GluR1 and GluR2 protein levels in the PVN in SHR and WKY rats. $\boldsymbol{A}$, Representative gel images show GluR1 and GluR2 protein levels in the plasma membrane and CVF in the PVN in SHR and WKY rats. Molecular weights are indicated on the right. $\boldsymbol{B}, \boldsymbol{C}$, Summary data show changes in the protein levels of GluR2 (B) and GluR1 ( $C$ ) subunits in the plasma membrane and CVF in the PVN in SHR ( $n=4$ samples) and WKY rats ( $n=4$ samples). For each sample, PVN tissues from three rats were pooled. Data presented as means \pm SEM. ${ }^{*} p<0.05$ compared with the WKY group.
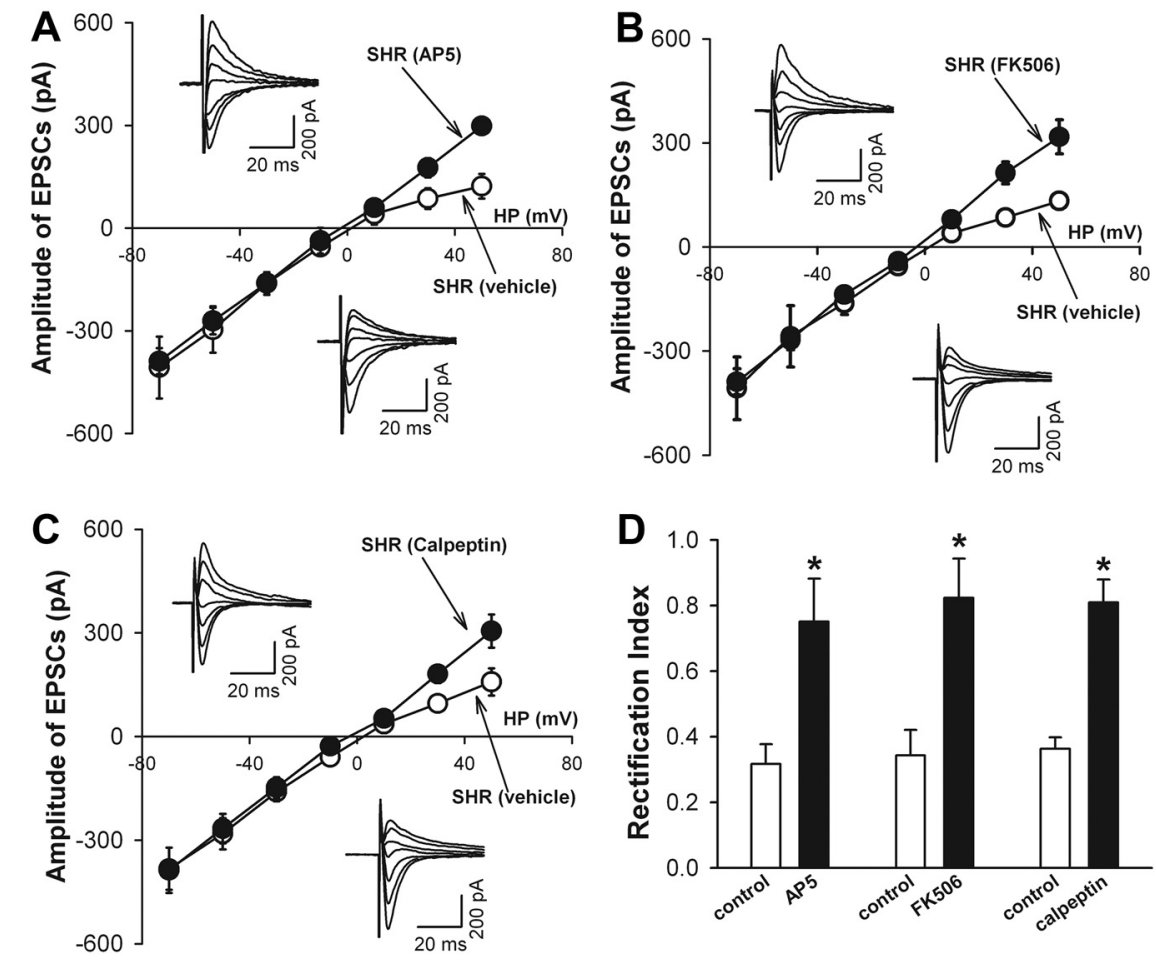

Figure 7. NMDA receptor-calpain-calcineurin signaling contributes to increased GluR2-lacking AMPAR activity of spinally projecting PVN neurons in SHR. $A, I-V$ curves and original current traces (inset) of AMPAR-EPSCs in labeled PVN neurons of SHR treated with AP-5 $(50 \mu \mathrm{M}, n=8)$ or vehicle $(n=10) . \boldsymbol{B}, I-V$ curves and original recordings (inset) of AMPAR-EPSCs in the PVN neurons of SHR treated with FK506 $(1 \mu \mathrm{m}, n=9)$ or vehicle $(n=7)$. C, I-V curves and representative traces (inset) of AMPAR-EPSCs in PVN neurons of SHR treated with calpeptin (100 $\mu \mathrm{m}, n=9)$ or vehicle $(n=8)$. HP, Holding potentials. D, Summary data show effects of treatment with AP-5 $(n=8)$, FK506 $(n=9)$, or calpeptin $(n=9)$ on the RI of AMPAR-EPSCs of labeled PVN neurons in SHR. Data are presented as mean \pm SEM. ${ }^{*} p<0.05$ compared with the vehicle control group. 
combination of GluR1, GluR3, and GluR4 (Oh and Derkach, 2005; Thiagarajan et al., 2005). Because of these functional properties, increased activity of GluR2-lacking AMPARs can increase excitatory synaptic strength (Terashima et al., 2004) and are involved in longterm potentiation consolidation in the hippocampus (Plant et al., 2006). Our results suggest that GluR2-lacking AMPARs play an important role in the control of the excitability of PVN presympathetic neuronal in this animal model of hypertension.

Because AMPARs that exhibit voltage-dependent blockade by intracellular polyamines are typically devoid of the GluR2 subunit (Bowie and Mayer, 1995; Koh et al., 1995), we reasoned that increased GluR2-lacking AMPAR activity of PVN neurons in SHR might be associated with increased GluR2 internalization. Consistent with this hypothesis, we found that the GluR2 protein amount in the plasma membrane in the PVN was significantly lower in SHR than in WKY rats. Also, the GluR2 protein level in the cytosolic vesicle fraction in the PVN was significantly higher in SHR than in WKY rats. However, GluR1 protein level in the plasma membrane and cytosolic vesicle fraction in the PVN did not differ significantly between WKY and SHR. Thus, our finding suggests that increased GluR2 internalization accounts for the inward rectification of AMPAR-EPSCs in PVN presympathetic neurons in this animal model of hypertension.

We found in this study that microinjection of the selective GluR2-lacking AMPAR blocker into the PVN significantly reduced ABP and sympathetic nerve discharges in SHR but not in WKY rats. This finding suggests that increased activity of GluR2lacking AMPARs in the PVN is important for the maintenance of elevated sympathetic outflow in SHR. It has been shown that neuronal activity can influence the trafficking of AMPARs at postsynaptic sites in cortical and hippocampus neurons (Schwarz et al., 2010). Because the excitability of PVN neurons is increased in SHR (Li and Pan, 2006; Li et al., 2008), we determined whether increased GluR2-lacking AMPAR activity results from elevated neuronal activity of PVN neurons in SHR. However, we observed that, after inhibiting neuronal activity with TTX, the $I-V$ relationship of AMPA currents of PVN neurons still displayed inward rectification at positive holding potentials in SHR. In addition, to determine whether increased activity of GluR2lacking AMPARs in the PVN is a secondary change to high blood pressure in SHR, we examined the $I-V$ relationship of AMPAREPSCs in spinally projecting PVN neurons after lowering ABP with CGx in SHR (Ye et al., 2011). We found that CGx effectively lowered ABP for at least 2 weeks in SHR. Nevertheless, inward rectification of AMPAR-EPSCs of PVN neurons still remained in these rats. Thus, increased GluR2-lacking AMPAR activity in the $\mathrm{PVN}$ is not caused by increased neuronal activity and high blood pressure but probably contributes to the development of hyperexcitability of PVN presympathetic neurons and hypertension.

The signaling mechanisms through which GluR2-lacking AMPAR activity is increased in the PVN in hypertension remain unclear. NMDA receptor-induced $\mathrm{Ca}^{2+}$ influx is involved in GluR2 internalization and synaptic plasticity in the hippocampus (Tigaret et al., 2006). The $\mathrm{Ca}^{2+}$-dependent calcineurin can dephosphorylate proteins involved in the endocytosis process and is involved in internalization of GluR2 induced by insulin and NMDA receptor stimulation (Slepnev et al., 1998; Lai et al., 1999; Beattie et al., 2000; Lin et al., 2000). Because increased glutamate release and NMDAR activity of PVN neurons are persistent in brain slices of SHR (Li et al., 2008; Ye et al., 2011), we hypothesized that augmented NMDA receptor activity contributes to increased GluR2-lacking AMPAR activity of PVN presympathetic neurons in hypertension. We found that blocking NMDA receptors with AP-5 eliminated the inward rectification of AMPAR-EPSCs of PVN neurons in SHR. Furthermore, we found that inhibition of calcineurin activity abolished inward rectification of AMPAR-EPSCs of spinally projecting PVN neurons in SHR. Our findings indicate that activation of NMDA receptors and calcineurin are involved in increased GluR2-lacking AMPAR activity in PVN presympathetic neurons in hypertension. It has been shown that calcineurin-dependent protein dephosphorylation is involved in GluR1 endocytosis in hippocampal neurons (Beattie et al., 2000; Biou et al., 2008). However, we found that there was no difference in the GluR1 membrane level in the PVN between WKY rats and SHR. It has been reported that calcineurin inhibition increases GluR2 phosphorylation in the striatum (Ahn and Choe, 2010). It is likely that calcineurin-dependent dephosphorylation can also influence GluR2 trafficking in PVN neurons in SHR. The amplitude of AMPAR-EPSCs at the resting membrane potential did not differ between WKY rats and SHR. Despite the decrease in the GluR2 membrane level, the membrane level of GluR1 was not altered in the PVN of SHR. Because GluR1-GluR3 subunits have been detected in the PVN (Eyigor et al., 2001), it is possible that the GluR3 may be increased at the membrane to maintain the AMPAR conductance of PVN neurons in SHR. In addition, activation of NMDA receptors induces $\mathrm{Ca}^{2+}$ influx to activate the $\mathrm{Ca}^{2+}$-dependent cysteine protease calpain (Adamec et al., 1998; Hewitt et al., 1998), which can convert calcineurin to a constitutively active form by cleaving the autoinhibitory domain in calcineurin A (Klee et al., 1998; Kim et al., 2002; Wu et al., 2004). In the present study, we found that inhibition of calpain eliminated the inward rectification of AMPAR-EPSCs of labeled PVN neurons in SHR. Therefore, it is likely that increased calpain activity couples the NMDA receptors to calcineurin and consequently induces GluR2 internalization in PVN presympathetic neurons in hypertension.

In summary, our study provides new information that the activity of GluR2-lacking AMPARs is increased and contributes to increased firing activity of spinally projecting PVN neurons in hypertension. Furthermore, NMDA receptor activation and subsequent stimulation of calpain and calcineurin lead to internalization of GluR2 subunit and increased activity of GluR2-lacking AMPARs in the PVN in hypertension. These findings provide new insights into the synaptic and signaling mechanisms underlying increased neuronal activity of PVN presympathetic neurons and sympathetic vasomotor tone in hypertension. This new information is important for our understanding of the mechanisms underlying the hypothalamic synaptic plasticity and development of neurogenic hypertension.

\section{References}

Adamec E, Beermann ML, Nixon RA (1998) Calpain I activation in rat hippocampal neurons in culture is NMDA receptor selective and not essential for excitotoxic cell death. Brain Res Mol Brain Res 54:35-48.

Ahn SM, Choe ES (2010) Alterations in GluR2 AMPA receptor phosphorylation at serine 880 following group I metabotropic glutamate receptor stimulation in the rat dorsal striatum. J Neurosci Res 88:992-999.

Allen AM (2002) Inhibition of the hypothalamic paraventricular nucleus in spontaneously hypertensive rats dramatically reduces sympathetic vasomotor tone. Hypertension 39:275-280.

Anderson EA, Sinkey CA, Lawton WJ, Mark AL (1989) Elevated sympathetic nerve activity in borderline hypertensive humans. Evidence from direct intraneural recordings. Hypertension 14:177-183.

Beattie EC, Carroll RC, Yu X, Morishita W, Yasuda H, von Zastrow M, Malenka RC (2000) Regulation of AMPA receptor endocytosis by a signaling mechanism shared with LTD. Nat Neurosci 3:1291-1300.

Biou V, Bhattacharyya S, Malenka RC (2008) Endocytosis and recycling of AMPA receptors lacking GluR2/3. Proc Natl Acad Sci U S A 105:1038-1043.

Blaschke M, Keller BU, Rivosecchi R, Hollmann M, Heinemann S, Konnerth A (1993) A single amino acid determines the subunit-specific spider 
toxin block of alpha-amino-3-hydroxy-5-methylisoxazole-4-propionate/ kainate receptor channels. Proc Natl Acad Sci U S A 90:6528-6532.

Bowie D, Mayer ML (1995) Inward rectification of both AMPA and kainate subtype glutamate receptors generated by polyamine-mediated ion channel block. Neuron 15:453-462.

Croall DE, DeMartino GN (1991) Calcium-activated neutral protease (calpain) system: structure, function, and regulation. Physiol Rev 71:813-847.

Cull-Candy S, Kelly L, Farrant M (2006) Regulation of $\mathrm{Ca}^{2+}$-permeable AMPA receptors: synaptic plasticity and beyond. Curr Opin Neurobiol 16:288-297.

Dingledine R, Borges K, Bowie D, Traynelis SF (1999) The glutamate receptor ion channels. Pharmacol Rev 51:7-61.

Donevan SD, Rogawski MA (1995) Intracellular polyamines mediate inward rectification of $\mathrm{Ca}^{2+}$-permeable alpha-amino-3-hydroxy-5-methyl-4isoxazolepropionic acid receptors. Proc Natl Acad Sci U S A 92:9298-9302.

Eilam R, Malach R, Bergmann F, Segal M (1991) Hypertension induced by hypothalamic transplantation from genetically hypertensive to normotensive rats. J Neurosci 11:401-411.

Esler M (1995) Sympathetic nervous system: contribution to human hypertension and related cardiovascular diseases. J Cardiovasc Pharmacol 26 [Suppl 2]:S24-S28.

Eyigor O, Centers A, Jennes L (2001) Distribution of ionotropic glutamate receptor subunit mRNAs in the rat hypothalamus. J Comp Neurol 434:101-124.

Hewitt KE, Lesiuk HJ, Tauskela JS, Morley P, Durkin JP (1998) Selective coupling of mu-calpain activation with the NMDA receptor is independent of translocation and autolysis in primary cortical neurons. J Neurosci Res 54:223-232.

Hollmann M, Hartley M, Heinemann S (1991) $\mathrm{Ca}^{2+}$ permeability of KAAMPA-gated glutamate receptor channels depends on subunit composition. Science 252:851-853.

Isaac JT, Ashby MC, McBain CJ (2007) The role of the GluR2 subunit in AMPA receptor function and synaptic plasticity. Neuron 54:859-871.

Judy WV, Watanabe AM, Henry DP, Besch HR Jr, Murphy WR, Hockel GM (1976) Sympathetic nerve activity: role in regulation of blood pressure in the spontaneously hypertensive rat. Circ Res 38:21-29.

Kamboj SK, Swanson GT, Cull-Candy SG (1995) Intracellular spermine confers rectification on rat calcium-permeable AMPA and kainate receptors. J Physiol 486:297-303.

Keller BU, Hollmann M, Heinemann S, Konnerth A (1992) Calcium influx through subunits GluR1/GluR3 of kainate/AMPA receptor channels is regulated by cAMP dependent protein kinase. EMBO J 11:891-896.

Kim MJ, Jo DG, Hong GS, Kim BJ, Lai M, Cho DH, Kim KW, Bandyopadhyay A, Hong YM, Kim DH, Cho C, Liu JO, Snyder SH, Jung YK (2002) Calpain-dependent cleavage of cain/cabin1 activates calcineurin to mediate calcium-triggered cell death. Proc Natl Acad Sci U S A 99:9870-9875.

Klee CB, Ren H, Wang X (1998) Regulation of the calmodulin-stimulated protein phosphatase, calcineurin. J Biol Chem 273:13367-13370.

Koh DS, Burnashev N, Jonas P (1995) Block of native $\mathrm{Ca}^{2+}$-permeable AMPA receptors in rat brain by intracellular polyamines generates double rectification. J Physiol 486:305-312.

Koike M, Ino M, Ozawa S (1997) Blocking effect of 1-naphthyl acetyl spermine on $\mathrm{Ca}^{2+}$-permeable AMPA receptors in cultured rat hippocampal neurons. Neurosci Res 29:27-36.

Lai MM, Hong JJ, Ruggiero AM, Burnett PE, Slepnev VI, De Camilli P, Snyder SH (1999) The calcineurin-dynamin 1 complex as a calcium sensor for synaptic vesicle endocytosis. J Biol Chem 274:25963-25966.

Li DP, Pan HL (2006) Plasticity of GABAergic control of hypothalamic presympathetic neurons in hypertension. Am J Physiol Heart Circ Physiol 290:H1110-H1119.

Li DP, Pan HL (2007) Glutamatergic inputs in the hypothalamic paraventricular nucleus maintain sympathetic vasomotor tone in hypertension. Hypertension 49:916-925.

Li DP, Chen SR, Pan HL (2003) Angiotensin II stimulates spinally projecting paraventricular neurons through presynaptic disinhibition. J Neurosci 23:5041-5049.

Li DP, Yang Q, Pan HM, Pan HL (2008) Pre- and postsynaptic plasticity underlying augmented glutamatergic inputs to hypothalamic presympathetic neurons in spontaneously hypertensive rats. J Physiol 586:1637-1647.

Lin JW, Ju W, Foster K, Lee SH, Ahmadian G, Wyszynski M, Wang YT, Sheng M (2000) Distinct molecular mechanisms and divergent endocytotic pathways of AMPA receptor internalization. Nat Neurosci 3:1282-1290.
Liu J (1993) FK506 and ciclosporin: molecular probes for studying intracellular signal transduction. Trends Pharmacol Sci 14:182-188.

Mayer ML, Armstrong N (2004) Structure and function of glutamate receptor ion channels. Annu Rev Physiol 66:161-181.

Molinari M, Carafoli E (1997) Calpain: a cytosolic proteinase active at the membranes. J Membr Biol 156:1-8.

Noh KM, Yokota H, Mashiko T, Castillo PE, Zukin RS, Bennett MV (2005) Blockade of calcium-permeable AMPA receptors protects hippocampal neurons against global ischemia-induced death. Proc Natl Acad Sci U S A 102:12230-12235.

Oh MC, Derkach VA (2005) Dominant role of the GluR2 subunit in regulation of AMPA receptors by CaMKII. Nat Neurosci 8:853-854.

Park JS, Voitenko N, Petralia RS, Guan X, Xu JT, Steinberg JP, Takamiya K, Sotnik A, Kopach O, Huganir RL, Tao YX (2009) Persistent inflammation induces GluR2 internalization via NMDA receptor-triggered PKC activation in dorsal horn neurons. J Neurosci 29:3206-3219.

Paxinos G, Watson C (1998) The rat brain in stereotaxic coordinates. San Diego: Academic

Plant K, Pelkey KA, Bortolotto ZA, Morita D, Terashima A, McBain CJ, Collingridge GL, Isaac JT (2006) Transient incorporation of native GluR2-lacking AMPA receptors during hippocampal long-term potentiation. Nat Neurosci 9:602-604.

Ranson RN, Motawei K, Pyner S, Coote JH (1998) The paraventricular nucleus of the hypothalamus sends efferents to the spinal cord of the rat that closely appose sympathetic preganglionic neurones projecting to the stellate ganglion. Exp Brain Res 120:164-172.

Schwarz LA, Hall BJ, Patrick GN (2010) Activity-dependent ubiquitination of GluAl mediates a distinct AMPA receptor endocytosis and sorting pathway. J Neurosci 30:16718-16729.

Shioda N, Moriguchi S, Shirasaki Y, Fukunaga K (2006) Generation of constitutively active calcineurin by calpain contributes to delayed neuronal death following mouse brain ischemia. J Neurochem 98:310-320.

Slepnev VI, Ochoa GC, Butler MH, Grabs D, De Camilli P (1998) Role of phosphorylation in regulation of the assembly of endocytic coat complexes. Science 281:821-824.

Swanson LW, Sawchenko PE (1983) Hypothalamic integration: organization of the paraventricular and supraoptic nuclei. Annu Rev Neurosci 6:269-324.

Terashima A, Cotton L, Dev KK, Meyer G, Zaman S, Duprat F, Henley JM, Collingridge GL, Isaac JT (2004) Regulation of synaptic strength and AMPA receptor subunit composition by PICK1. J Neurosci 24:5381-5390.

Thiagarajan TC, Lindskog M, Tsien RW (2005) Adaptation to synaptic inactivity in hippocampal neurons. Neuron 47:725-737.

Tigaret CM, Thalhammer A, Rast GF, Specht CG, Auberson YP, Stewart MG, Schoepfer R (2006) Subunit dependencies of N-methyl-D-aspartate (NMDA) receptor-induced alpha-amino-3-hydroxy-5-methyl-4-isoxazolepropionic acid (AMPA) receptor internalization. Mol Pharmacol 69:1251-1259.

Tsujinaka T, Kajiwara Y, Kambayashi J, Sakon M, Higuchi N, Tanaka T, Mori $\mathrm{T}$ (1988) Synthesis of a new cell penetrating calpain inhibitor (calpeptin). Biochem Biophys Res Commun 153:1201-1208.

Victor RG, Shafiq MM (2008) Sympathetic neural mechanisms in human hypertension. Curr Hypertens Rep 10:241-247.

Vikman KS, Rycroft BK, Christie MJ (2008) Switch to $\mathrm{Ca}^{2+}$-permeable AMPA and reduced NR2B NMDA receptor-mediated neurotransmission at dorsal horn nociceptive synapses during inflammatory pain in the rat. J Physiol 586:515-527.

Wu HY, Tomizawa K, Oda Y, Wei FY, Lu YF, Matsushita M, Li ST, Moriwaki A, Matsui H (2004) Critical role of calpain-mediated cleavage of calcineurin in excitotoxic neurodegeneration. J Biol Chem 279:4929-4940.

Yamashita H, Inenaga K, Koizumi K (1984) Possible projections from regions of paraventricular and supraoptic nuclei to the spinal cord: electrophysiological studies. Brain Res 296:373-378.

Yamori Y, Okamoto K (1969) Hypothalamic tonic regulation of blood pressure in spontaneously hypertensive rats. Jpn Circ J 33:509-519.

Ye ZY, Li DP, Li L, Pan HL (2011) Protein kinase CK2 increases glutamatergic input in the hypothalamus and sympathetic vasomotor tone in hypertension. J Neurosci 31:8271-8279.

Zahner MR, Pan HL (2005) Role of paraventricular nucleus in the cardiogenic sympathetic reflex in rats. Am J Physiol Regul Integr Comp Physiol 288:R420-R426. 